Brazilian Journal

of Chemical

ISSN 0104-6632

Engineering

Printed in Brazil

Vol. 19, No. 02, pp. 195 - 206, April - June 2002

\title{
APPLICATION OF THE RPN METHODOLOGY FOR QUANTIFICATION OF THE OPERABILITY OF THE QUADRUPLE-TANK PROCESS
}

\author{
J.O.Trierweiler \\ Laboratory of Process Control and Integration (LACIP), Department of Chemical Engineering, \\ Federal University of Rio Grande do Sul (UFRGS), Rua Marechal Floriano 501, \\ CEP 90020-061, Porto Alegre - RS, Brazil \\ E-mail: jorge@enq.ufrgs.br
}

(Received: October 23, 2001; Accepted: February 8, 2002)

\begin{abstract}
The RPN indicates how potentially difficult it is for a given system to achieve the desired performance robustly. It reflects both the attainable performance of a system and its degree of directionality. Two new indices, RPN ratio and RPN difference are introduced to quantify how realizable a given desired performance can be. The predictions made by RPN are verified by closed-loop simulations. These indices are applied to quantify the IO-controllability of the quadruple-tank process.

Keywords: controllability measures, RPN, the quadruple-tank system, controller design.
\end{abstract}

\section{INTRODUCTION}

Quantitative input-output controllability measures are key ingredients of a systematic control structure design (CSD) procedure. Many different aspects (e.g., model uncertainties, nonlinearity of the process, input saturation, interactions between the control loops) must be taken into account. In Trierweiler (1997) and Trierweiler and Engell (1997a) the Robust Performance Number (RPN) and the Robust Performance Number with constant scalings $\left(\mathrm{RPN}_{\mathrm{LR}}\right)$ were introduced to characterize the IO-controllability of a system. Here two new indices based on the RPN concept are proposed: RPN ratio and RPN difference. These new indices allow us to quantify how far the attainable performance is from the desired one.

In this paper, we apply these indices to analyze the quadruple-tank process proposed by Johansson (2000). The quadruple-tank process is a laboratory process that consists of four interconnected water tanks. The linearized dynamic model of the system has a real multivariable transmission zero which can change its sign depending on operating conditions. In this way, the quadruple-tank process is ideal for illustrating many concepts in multivariable control, particularly performance limitations due to multivariable RHP zeros. In the paper, both nonminimum- and minimum-phase operating points are analyzed and systematically compared using the RPN concept. The paper also shows how the RPN methodology can be applied to controller design.

The paper is structured as follows: in section 2, the RPN concept and the new indices are introduced. In section 3, the quadruple-tank process is described. In section 4, the IO-controllability analysis is performed using RPN, $\mathrm{RPN}_{\mathrm{LR}}$, RPN ratio, and RPN difference indices. In section 5, the predictions based on the RPN concept are confirmed by closed-loop simulations.

RPN - A CRITERION FOR CONTROL 


\section{STRUCTURE SELECTION}

The Robust Performance Number (RPN) was introduced in Trierweiler (1997) and Trierweiler and Engell (1997a) as a measure to characterize the IOcontrollability of a system. The RPN indicates how potentially difficult it is for a given system to achieve the desired performance robustly. The RPN is influenced by both the desired performance of a system and its degree of directionality.

\section{The Robust Performance Number}

Definiton: The Robust Performance Number ( RPN, $\Gamma$ ) of a multivariable plant with transfer matrix $G(s)$ is defined as

$$
\mathrm{RPN} \stackrel{\Delta}{=} \Gamma_{\text {sup }}(\mathrm{G}, \mathrm{T}, \omega)=\sup _{\omega \in \mathrm{R}}\{\Gamma(\mathrm{G}, \mathrm{T}, \omega)\}
$$

$$
\begin{aligned}
& \Gamma(\mathrm{G}, \mathrm{T}, \omega) \stackrel{\Delta}{=} \\
& \stackrel{\Delta}{=} \sqrt{\bar{\sigma}([\mathrm{I}-\mathrm{T}(\mathrm{j} \omega)] \mathrm{T}(\mathrm{j} \omega))\left(\gamma^{*}(\mathrm{G}(\mathrm{j} \omega))+\frac{1}{\gamma^{*}(\mathrm{G}(\mathrm{j} \omega))}\right)}
\end{aligned}
$$

where $\gamma^{*}(\mathrm{G}(\mathrm{j} \omega))$ is the minimized condition number of $\mathrm{G}(\mathrm{j} \omega)$ and $\bar{\sigma}([\mathrm{I}-\mathrm{T}] \mathrm{T})$ is the maximal singular value of the transfer function $[\mathrm{I}-\mathrm{T}] \mathrm{T} . \mathrm{T}$ is the (attainable) desired output complementary sensitivity function, which is determined for the nominal model $\mathrm{G}(\mathrm{s})$.

The minimized condition number, $\gamma^{*}(\mathrm{G}(\mathrm{j} \omega)$, is defined by $\gamma^{*}(G(j \omega)) \stackrel{\Delta}{=} \min _{L, R} \gamma(L G(j \omega) R)$, where $L$ and $\mathrm{R}$ are real, diagonal, and nonsingular scaling matrices and $\gamma$ is the Euclidean condition number. The Euclidean condition number $\gamma$ of a complex matrix $\mathrm{M}$ is defined as the ratio between the maximal and minimal singular values, i.e.,

$\gamma(\mathrm{M}) \stackrel{\Delta}{=} \frac{\bar{\sigma}(\mathrm{M})}{\underline{\sigma}(\mathrm{M})}$.

The RPN consists of two factors:

1) $\bar{\sigma}([\mathrm{I}-\mathrm{T}] \mathrm{T})$. This term acts as a weighting function and emphasizes the more important region (i.e., the crossover frequency range) for robust stability and robust performance relative to the low and high frequency regions that are less important for feedback control. For example, a system can have a high degree of uncertainty at low frequencies, but nevertheless show no stability and performance problems. This fact is automatically taken into account by the function $\bar{\sigma}([\mathrm{I}-\mathrm{T}] \mathrm{T})$, which has its peak value in the crossover frequency range. The choice of $\mathrm{T}$ depends on the desired closed-loop bandwidth, sensor noise, input constraints, and in particular the nonminimum-phase part of G, i.e., RHP zeros, RHP poles, and pure time delays.

2) $\gamma *(\mathrm{G})+1 / \gamma *(\mathrm{G})$. The origin of this term is the result of computation of the robust performance (RP) of inverse-based controllers (see Trierweiler and Engell, 1997a).

The RPN is a measure of how potentially difficult it is for a given system to achieve the desired performance robustly. The easiest way to design a controller is to use the inverse of process model. An inverse-based controller will have potentially good performance robustness only when the RPN is small. As inverse-based controllers are simple and effective, it can be concluded that a good control structure selection is one with a small $(<5)$ RPN (Trierweiler and Engell, 2000).

\section{RPN-Scaling Procedure}

The scaling of the transfer matrix is very important for the correct analysis of the controllability of a system and for controller design. In the definition of $\gamma^{*}(\mathrm{G}(\mathrm{j} \omega)), \mathrm{L}$ and $\mathrm{R}$ are frequency dependent; however, in the design stage $\mathrm{L}$ and $\mathrm{R}$ are usually constant. The following procedure based on the RPN is recommended for use in optimal scaling of a system, G.

\section{RPN-scaling procedure:}

1. Determine the frequency, $\omega_{\text {sup }}$, where $\Gamma(\mathrm{G}, \mathrm{T}, \omega)$ achieves its maximal value.

2. Calculate the scaling matrices, $\mathrm{L}_{\mathrm{S}}$ and $\mathrm{R}_{\mathrm{S}}$, such that $\gamma\left(\mathrm{L}_{\mathrm{S}} \mathrm{G}\left(\mathrm{j} \omega_{\text {sup }}\right) \mathrm{R}_{\mathrm{S}}\right)$ achieves its minimal value, $\gamma^{*}\left(\mathrm{G}\left(\mathrm{j} \omega_{\text {sup }}\right)\right)$.

3. Scale the system with the scaling matrices, $L_{S}$ and $R_{S}$, i.e., $G_{S}(s)=L_{S} G(s) R_{S}$

Analysis and controller design should then be performed with the scaled system, $\mathrm{G}_{\mathrm{S}}$.

RPN with Constant Scalings 
Definition: The robust performance number with constant scalings ( $\mathrm{RPN}_{\mathrm{LR}}, \Gamma_{\mathrm{LR}}$ ) of a multivariable plant with transfer matrix $G(s)$ is defined as

$$
\begin{gathered}
\gamma(j \omega)^{\Delta}=\gamma\left(L_{s} G(j \omega) R_{s}\right) \\
\Gamma_{L R}(G, T, \omega)=\sqrt[\Delta]{\bar{\sigma}([I-T(j \omega)] T(j \omega))\left(\gamma(j \omega)+\frac{1}{\gamma(j \omega)}\right)} \\
\operatorname{RPN}_{L R}=\sup _{\omega \in R}\left\{\Gamma_{L R}(G, T, \omega)\right\}
\end{gathered}
$$

where $L_{S}$ and $R_{S}$ are fixed scaling matrices corresponding to the scaling matrices that make $\gamma$ $\left(L_{S} G\left(j \omega_{\text {sup }}\right) R_{S}\right)$ minimal, i.e., $L_{S}$ and $R_{S}$ are the scaling matrices calculated by the RPN-scaling procedure.

\section{Attainable Performance}

In this section, it is discussed how the attainable closed-loop performance can be characterized for systems with RHP transmission zeros.

\section{(a) Specification of the Desired Performance}

We specify the desired performance by the (output) complementary sensitivity function, $\mathrm{T}$, which relates the reference signal, $r$, and the output signal, $y$, in the one degree of freedom (DOF) control configuration ( see Fig. 1 ). For the SISO case, specifications such as settling time, rise time, maximal overshoot, and steady-state error can be mapped into the choice of a transfer function of the form

$$
\mathrm{T}_{\mathrm{d}}=\frac{\Delta}{\left(\frac{\mathrm{s}}{\omega_{\mathrm{n}}}\right)^{2}+2 \zeta \frac{\mathrm{s}}{\omega_{\mathrm{n}}}+1}
$$

where $\varepsilon_{\infty}$ is the tolerated offset (steady-state error). The parameters of equation (3), $\omega_{n}$ (undamped natural frequency) and $\zeta$ (damping ratio), can be easily calculated from the time-domain specifications.

For the MIMO case, a straightforward extension of this specification is to prescribe a decoupled response with possibly different parameters for each output, i.e., $T_{d}=\operatorname{diag}\left(T_{d, 1}, \ldots, T_{d, n o}\right)$, where each $\mathrm{T}_{\mathrm{d}, \mathrm{i}}$ corresponds to a SISO time-domain specification.

\section{(b) RHP-Zero Constraint and Factorization}

If $\mathrm{G}(\mathrm{s})$ has a RHP zero at $\mathrm{z}$ with output direction $y_{z}$, then for internal stability of the feedback system the controller must not cancel the RHP zero. Thus $\mathrm{L}=\mathrm{GK}$ must also have a RHP zero in the same direction as $\mathrm{G}$, i.e., $\mathrm{y}_{\mathrm{z}}^{\mathrm{H}} \mathrm{G}(\mathrm{z})=0 \Rightarrow \mathrm{y}_{\mathrm{z}}{ }^{\mathrm{H}} \mathrm{G}(\mathrm{z}) \mathrm{K}(\mathrm{z})=0$. It follows from $\mathrm{T}=\mathrm{LS}$ that the interpolation constraints

$\mathrm{y}_{\mathrm{Z}}^{\mathrm{H}} \mathrm{T}(\mathrm{z})=0 ; \mathrm{y}_{\mathrm{Z}}^{\mathrm{H}} \mathrm{S}(\mathrm{z})=\mathrm{y}_{\mathrm{Z}}^{\mathrm{H}}$

must be satisfied.

When the plant $\mathrm{G}(\mathrm{s})$ is asymptotically stable and has at least as many inputs as outputs, $G(s)$ can be factored as $\mathrm{G}(\mathrm{s})=\mathrm{B}_{\mathrm{O}, \mathrm{z}}(\mathrm{s}) \mathrm{G}_{\mathrm{m}}(\mathrm{s})$. The possible closedloop transfer functions $\mathrm{T}$ can then be factored to satisfy the interpolation constraint (4) as

$$
\mathrm{T}(\mathrm{s})=\mathrm{B}_{\mathrm{O}, \mathrm{z}}(\mathrm{s}) \mathrm{B}_{\mathrm{O}, \mathrm{z}}^{\dagger}(0) \mathrm{T}_{\mathrm{d}}(\mathrm{s})
$$

where $T_{d}(s)$ is the ideal desired closed-loop transfer function and $\mathrm{B}_{\mathrm{O}, \mathrm{Z}}(\mathrm{s})$ is the output Blaschke factorization for the zeros (for the definition of the Blaschke factorization and an algorithm to calculate it, see, e.g., Havre and Skogestad (1996) or Trierweiler (1997)). $\mathrm{B}_{\mathrm{O}, \mathrm{z}}{ }^{\dagger}$ denotes the pseudo-inverse of $\mathrm{B}_{\mathrm{O}, \mathrm{z}}$, and $\mathrm{B}_{\mathrm{O}, \mathrm{z}}(0) \mathrm{B}_{\mathrm{O}, \mathrm{z}}^{\dagger}(0)=\mathrm{I}$. It is easy to verify that (5) implies (4).

$\mathrm{T}(\mathrm{s})$ is different from the original desired transfer function $T_{d}(s)$, but has the same singular values. The factor $\mathrm{B}_{\mathrm{O}, \mathrm{z}}^{\dagger}(0)$ ensures that $\mathrm{T}(0)=\mathrm{T}_{\mathrm{d}}(0)$ so that the steady-state characteristics ( usually $\mathrm{T}_{\mathrm{d}}(0)=\mathrm{I}$ ) are preserved.

\section{(c) Remarks about the Blaschke Factorization:}

1) An alternative to the Blaschke factorization is to solve a standard optimal LQ control problem. This procedure is implemented in Chiang and Safonov (1992, see functions iofr and iofc). This inner-outer factorization requires system $\mathrm{G}(\mathrm{s})$ to be stable and to have no j $\omega$-axis or infinite poles or transmission zeros. In particular, D must have full rank. This means that for stable strictly proper systems replacing the matrix $D$ by $D_{\varepsilon}=\varepsilon I$ is necessary if we want to apply this factorization. Therefore, we prefer not to use this method and consequently it is not presented here. The interested reader will find further discussion and references to this procedure in Chiang 
and Safonov (1992).

2) For complex RHP zeros, the corresponding Blaschke factorization assumes a complex statespace model realization (Havre and Skogestad, 1996). Since the RPN analysis is based on the frequency response, this kind of representation does not impose any kind of limitation on the system analysis.

\section{Minimum Possible RPN ( $\left.\operatorname{RPN}_{\text {MIN }}\right)$}

When the system has a strong nonminimumphase behavior (e.g., RHP zero close to origin, large pure time delays), the attainable and the desired performances can be considerably different. Therefore, it is interesting to know the minimum possible RPN for a given desired performance. It can be calculated as follows:

$$
\begin{aligned}
\Gamma_{\mathrm{MIN}}\left(\mathrm{T}_{\mathrm{d}}, \omega\right) \stackrel{\Delta}{=} \sqrt{\bar{\sigma}\left(\left[\mathrm{I}-\mathrm{T}_{\mathrm{d}}(\mathrm{j} \omega)\right] \mathrm{T}_{\mathrm{d}}(\mathrm{j} \omega)\right) \times 2} \\
\operatorname{RPN}_{\mathrm{MIN}} \stackrel{\Delta}{=} \sup _{\omega}\left\{\Gamma_{\text {MIN }}\left(\mathrm{T}_{\mathrm{d}}, \omega\right)\right\}
\end{aligned}
$$

Note that RPN $\mathrm{N}_{\mathrm{MIN}}$ and $\Gamma_{\mathrm{MIN}}$ are only a function of the desired performance, $T_{d}$. The minimum possible condition number for any system is $\gamma^{*}(G(j \omega))=1$; thus the minimum possible value for $\gamma *(\mathrm{G})+1 / \gamma *(\mathrm{G})$ is 2 . This value is substituted into equation (1) and is used as the basis for the definition of RPN $\mathrm{MIN}_{\text {. }}$

Figure 2 shows an example of RPN, RPN $\mathrm{LR}_{\mathrm{L}}$, and $\mathrm{RPN}_{\mathrm{MIN}}$ plots. The larger the difference between RPN and $\mathrm{RPN}_{\mathrm{MIN}}$ plots, the more unrealizable the desired performance.

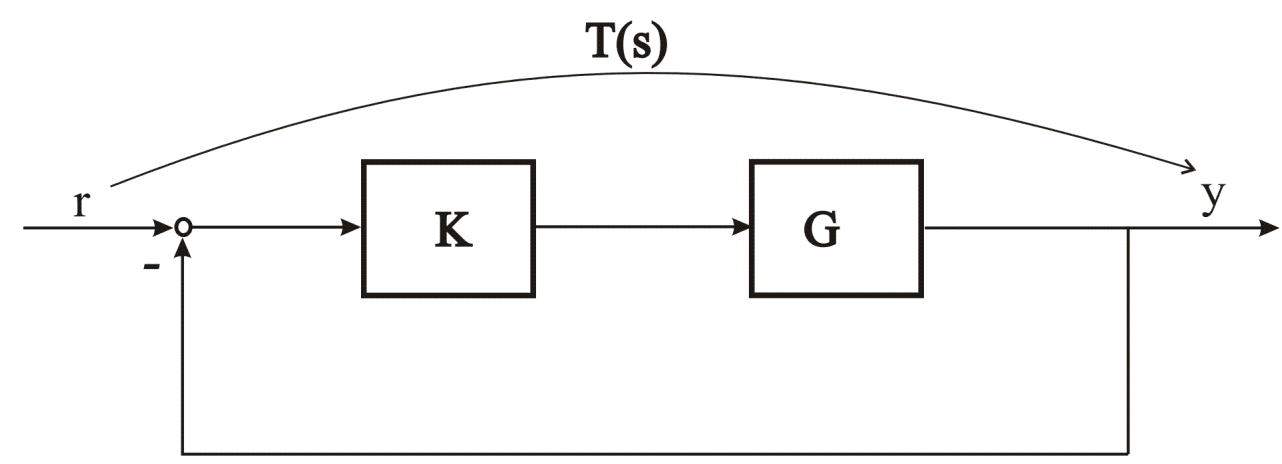

Figure 1: Standard feedback configuration

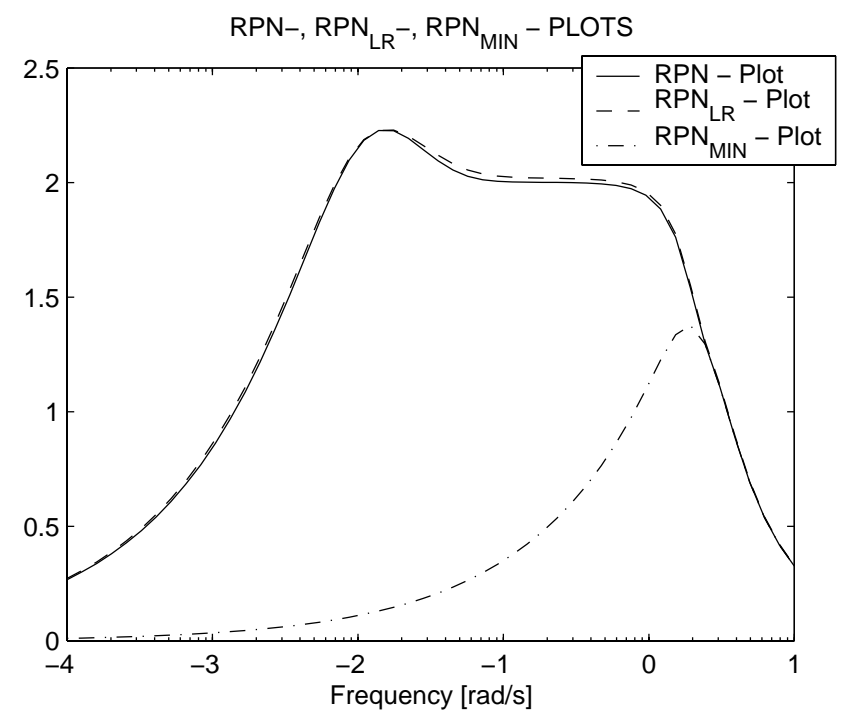

Figure 2: An example of RPN plot (solid line), $R P N_{L R}$ plot (dashed line), and $R P N_{M I N}$ plot (dashdot line). Note that the frequency is on a logarithmic scale so that -4 should be understood as $10^{-4}$.

\section{RPN Ratio and RPN Difference}


If the areas under the $\mathrm{RPN}_{\mathrm{MIN}}$ and RPN curves are calculated, i.e.,

$\mathrm{A}_{\mathrm{MIN}} \stackrel{\Delta}{=} \int_{\omega_{\min }}^{\omega_{\max }} \Gamma_{\mathrm{MIN}}\left(\mathrm{T}_{\mathrm{d}}, \omega\right) \mathrm{d} \log \omega$

$\mathrm{A} \stackrel{\Delta}{=} \int_{\omega_{\min }}^{\omega_{\max }} \Gamma(\mathrm{G}, \mathrm{T}, \omega) \mathrm{d} \log \omega$

it is easy to measure how far the curves are from each other. Based on these areas, the RPN ratio $\left(\mathrm{RPN}_{\mathrm{RATIO}}\right)$ and $\mathrm{RPN}$ difference $\left(\mathrm{RPN}_{\mathrm{DIFF}}\right)$ are defined as follows:

$$
\begin{aligned}
& \mathrm{RPN}_{\text {RATIO }}=\frac{\mathrm{A}}{\mathrm{A}_{\mathrm{MIN}}} \\
& \mathrm{RPN}_{\text {DIFF }}=\mathrm{A}-\mathrm{A}_{\mathrm{MIN}}
\end{aligned}
$$

Figure 3 gives a graphical interpretation of areas $\mathrm{A}_{\mathrm{MIN}}$ and $\mathrm{A}$. Note that the areas were calculated for a given frequency range, $\left[\omega_{\min }, \omega_{\max }\right]$, on a logarithmic scale. The frequency range must be large enough to capture the important region. When RPN $\mathrm{RATIO}_{\text {and }}$ $\mathrm{RPN}_{\mathrm{DIFF}}$ are used as relative measures, a simple finite interval can be used. But if an absolute measurement is required, then $\omega_{\min }$ and $\omega_{\max }$ must tend to 0 and $\propto$, respectively.

\section{CASE STUDY: THE QUADRUPLE - TANK PROCESS}

\section{Process Description}

The quadruple-tank process (see Figure 4) is a laboratory process that consists of four interconnected water tanks. The linearized dynamic model of the system has a real multivariable zero, whose sign can be changed depending on operating conditions. In this way, the quadruple-tank process is ideal for illustrating many concepts in multivariable control, particularly performance limitations due to multivariable RHP zeros. The location and the direction of zero have an appealing physical interpretation. The target is to control the level in the lower two tanks with the inlet flowrates, $F_{1}$ and $F_{2}$.

\section{Process Model}

The process model consists of the mass balance around each tank and is given by

$$
\begin{aligned}
& \mathrm{A}_{1} \frac{\mathrm{dh}_{1}}{\mathrm{dt}}=\mathrm{x}_{1} \cdot \mathrm{F}_{1}+\mathrm{R}_{3} \sqrt{\mathrm{h}_{3}}-\mathrm{R}_{1} \sqrt{\mathrm{h}_{1}} \\
& \mathrm{~A}_{2} \frac{\mathrm{dh}_{2}}{\mathrm{dt}}=\mathrm{x}_{2} \cdot \mathrm{F}_{2}+\mathrm{R}_{4} \sqrt{\mathrm{h}_{4}}-\mathrm{R}_{2} \sqrt{\mathrm{h}_{2}} \\
& \mathrm{~A}_{3} \frac{\mathrm{dh}_{3}}{\mathrm{dt}}=\left(1-\mathrm{x}_{2}\right) \cdot \mathrm{F}_{2}-\mathrm{R}_{3} \sqrt{\mathrm{h}_{3}} \\
& \mathrm{~A}_{4} \frac{\mathrm{dh}}{\mathrm{dt}}=\left(1-\mathrm{x}_{1}\right) \cdot \mathrm{F}_{1}-\mathrm{R}_{4} \sqrt{\mathrm{h}_{4}}
\end{aligned}
$$

where $A_{i}$ is the cross-section area of Tank $i, R_{i}$ is the outlet flow coefficient of Tank $i, h_{i}$ is the water level of Tank $i, F_{1}$ and $F_{2}$ are the manipulated inlet flowrates and $\mathrm{x}_{1}$ and $\mathrm{x}_{2}$ are the valve distribution flow factors $0 \leq \mathrm{x}_{\mathrm{i}} \leq 1$.

The parameters used in this work are basically the same as those in Johansson (2000) and are given by $\mathrm{A}_{1}=\mathrm{A}_{3}=28 \mathrm{~cm}^{2}, \mathrm{~A}_{2}=\mathrm{A}_{4}=32 \mathrm{~cm}^{2}$, $\mathrm{R}_{1}=\mathrm{R}_{3}=3.145 \mathrm{~cm}^{2.5} / \mathrm{s}$ and $\mathrm{R}_{2}=\mathrm{R}_{4}=2.525 \mathrm{~cm}^{2.5} / \mathrm{s}$.

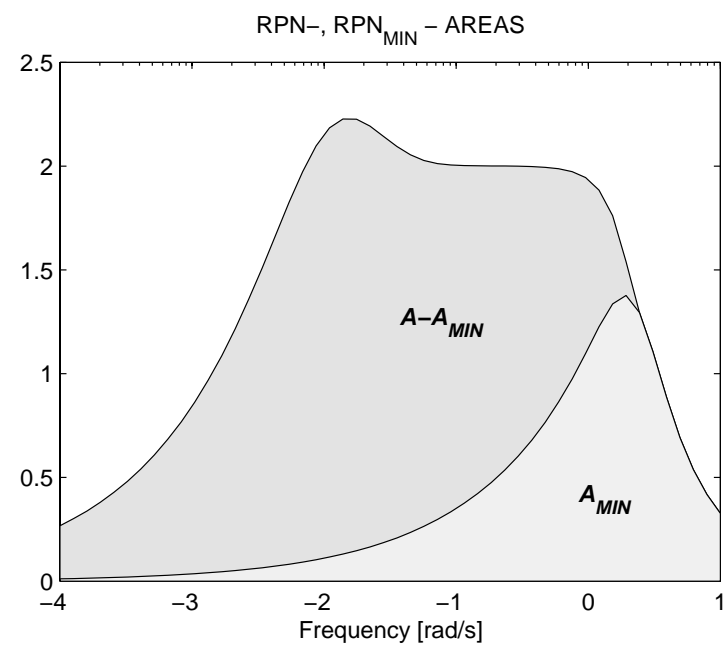

Figure 3: Schematic representation of $A_{M I N}$ and $A-A_{M I N}$. Note that the frequency is on a logarithmic scale so that -4 should be understood as $10^{-4}$. 


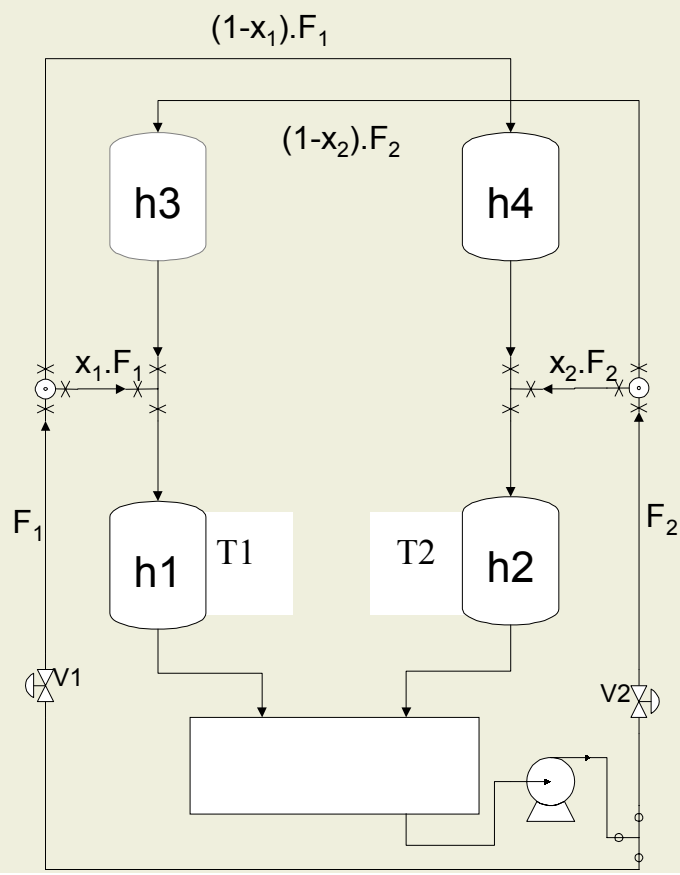

Figure 4: Schematic diagram of the quadruple-tank process. The water levels in Tank 1 and Tank 2 are controlled by the flow rates $F_{1}$ and $F_{2}$.

\section{Operating Points}

The quadruple-tank process is studied at a minimum-phase operating point (MOP) and at a nonminimum-phase operating point (NMOP), due to the presence of the RHP transmission zero. Table 1 summarizes the operating conditions of MOP and NMOP. Note that the main difference between the OPs is the valve distribution flow factors, $\mathrm{x}_{1}$ and $\mathrm{x}_{2}$, which are responsible for the difference in $h_{3}$ and $h_{4}$ levels. All other variables are almost the same for both OPs.

\section{RPN ANALYSIS FOR THE QUADRUPLE} TANK

\section{RHP Zero and RGA}

Johansson (2000) shows that the quadruple-tank system always has two transmission zeros, whose locations can be classified based on the $\mathrm{x}_{1}+\mathrm{x}_{2}$ value. When $0<\mathrm{x}_{1}+\mathrm{x}_{2}<1$, one of the transmission zeros is located in RHP. For the case where $\mathrm{x}_{1}+\mathrm{x}_{2}=1$, the system has a transmission zero at the origin, whereas for $1<\mathrm{x}_{1}+\mathrm{x}_{2}<2$ no RHP zero occurs. Table 2 shows the RHP zero for both OPs. For NMOP, the input zero direction, $\mathrm{u}_{\mathrm{Z}}$, and output zero direction, $y_{z}$, were also included in the table. The steady-state RGA (see Table 2) clearly shows that the pairing used for MOP (i.e., $\left(\mathrm{F}_{1}\right.$, $\left.\mathrm{h}_{1}\right)$ and $\left.\left(\mathrm{F}_{2}, \mathrm{~h}_{2}\right)\right)$ should not be applied to NMOP.

Table 1: Definition of the Operating Points

\begin{tabular}{|l|rr|rr|}
\hline Variables & \multicolumn{2}{|c|}{ MOP } & \multicolumn{2}{c|}{ NMOP } \\
\hline $\mathrm{h}_{1}, \mathrm{~h}_{2}[\mathrm{~cm}]$ & 12.26, & 12.78 & 12.44, & 13.16 \\
$\mathrm{~h}_{3}, \mathrm{~h}_{4}[\mathrm{~cm}]$ & 1.63, & 1.41 & 4.73, & 4.99 \\
$\mathrm{~F}_{1}, \mathrm{~F}_{2}\left[\mathrm{~cm}^{3} / \mathrm{s}\right]$ & 9.99, & 10.05 & 9.89, & 10.36 \\
$\mathrm{x}_{1}, \mathrm{x}_{2}[-]$ & 0.70, & 0.60 & 0.43, & 0.34 \\
\hline
\end{tabular}


Table 2: RHP zero and RGA

\begin{tabular}{|l|c|c|}
\hline & MOP & NMOP \\
\hline RHP zero & none & 0.0128 \\
RHP zero input direction & - & $\mathrm{u}_{\mathrm{z}}=\left[\begin{array}{c}0.7326 \\
-0.6806\end{array}\right]$ \\
RHP zero output direction & - & $\mathrm{yz}=\left[\begin{array}{c}-0.7743 \\
0.6329\end{array}\right]$ \\
\hline RGA(0) & {$\left[\begin{array}{cc}1.4 & -0.4 \\
-0.4 & 1.4\end{array}\right]$} & {$\left[\begin{array}{cc}-0.64 & 1.64 \\
1.64 & -0.64\end{array}\right]$} \\
\hline
\end{tabular}

\section{Dynamic RGA and Minimized Condition Number}

The transfer matrices for MOP and NMOP are respectively given by

$$
\begin{aligned}
& \mathrm{G}_{\mathrm{M}}(\mathrm{s})=\left[\begin{array}{cc}
\frac{4.89}{62 \mathrm{~s}+1} & \frac{2.93}{(23 \mathrm{~s}+1)(62 \mathrm{~s}+1)} \\
\frac{2.67}{(30 \mathrm{~s}+1)(90 \mathrm{~s}+1)} & \frac{5.59}{90 \mathrm{~s}+1}
\end{array}\right] \\
& \mathrm{G}_{\mathrm{NM}}(\mathrm{s})=\left[\begin{array}{cc}
\frac{3.03}{63 \mathrm{~s}+1} & \frac{4.87}{(39 \mathrm{~s}+1)(63 \mathrm{~s}+1)} \\
\frac{5.14}{(56 \mathrm{~s}+1)(91 \mathrm{~s}+1)} & \frac{3.22}{91 \mathrm{~s}+1}
\end{array}\right]
\end{aligned}
$$

Here the inputs are $\left(\mathrm{F}_{1}, \mathrm{~F}_{2}\right)$ and $\left(\mathrm{h}_{1}, \mathrm{~h}_{2}\right)$. Using these transfer matrices the minimum condition number and the element $(1,1)$ of the dynamic RGA were calculated. These results are shown in Figure 5. Note that for MOP the interaction disappears at high frequencies. This means that if the controller can be fast tuned, the control loops will behave like a completely non interacting system. For NMOP, the pairing $\left(\mathrm{F}_{1}, \mathrm{~h}_{2}\right)$ and $\left(\mathrm{F}_{2}, \mathrm{~h}_{1}\right)$ was used in the calculation. Note that the interaction pattern changes at around a frequency of $10^{-2} \mathrm{rad} / \mathrm{s}$. At low frequencies the best pairing is $\left(\mathrm{F}_{1}, \mathrm{~h}_{2}\right)$, but for high frequencies the pairing $\left(\mathrm{F}_{1}, \mathrm{~h}_{1}\right)$ will be much better. The minimum condition number shows that both OPs are well conditioned, especially at high frequencies.
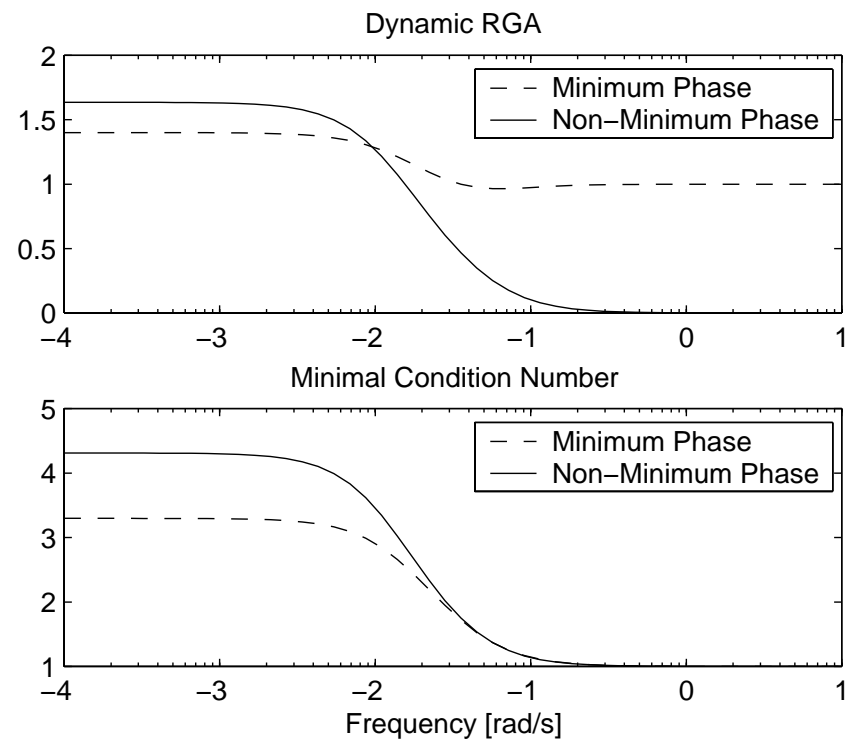

Figure 5: Dynamic RGA and Minimal Condition Number for MOP and NMOP

\section{RPN Analysis}


Table 3 shows the values of RPN, RPN $\mathrm{RATIO}_{\text {, and }}$ $\mathrm{RPN}_{\mathrm{DIFF}}$ calculated for MOP using several rise times and $5 \%$ overshoot. The corresponding RPN, RPN $\mathrm{LR}_{\mathrm{L}}$ and $\mathrm{RPN}_{\mathrm{MIN}}$ plots are shown in Figure 6. Based on these results, it can be concluded that for MOP the faster the closed loop, the better the system performance. The closed-loop response is only limited by saturation of manipulated variables.

Similarly to Table 3, Table 4 shows the values of RPN, RPN $N_{\text {RATIO }}$, and $\mathrm{RPN}_{\mathrm{DIFF}}$ calculated for NMOP using several rise times and 5\% overshoot. The corresponding RPN, $\mathrm{RPN}_{\mathrm{LR}}$ and $\mathrm{RPN}_{\mathrm{MIN}}$ plots are shown in Figure 7. Based on these results, it can be concluded that for NMOP the faster the closed loop, the more unrealizable the desired performance. Here, the closed-loop performance is limited by the RHP zero at 0.0128 . Note that all the peaks of RPN curves are at around a frequency equal to the RHP zero, i.e., $\omega=0.0128$. If the peak of the desired performance (i.e., peak of $\left.\bar{\sigma}\left(\left[I-T_{d}\right] T_{d}\right)\right)$ is above this frequency, the RPN curve shows a flat region up to the peak of the desired performance.

Table 3: RPN indices for MOP

\begin{tabular}{|l|l|l|l|}
\hline \multicolumn{1}{|c|}{$\begin{array}{c}\text { Rise } \\
\text { Time }[\mathbf{s}]\end{array}$} & \multicolumn{1}{|c|}{ RPN } & \multicolumn{1}{|c|}{ RPN $_{\text {RATIO }}$} & RPN $_{\text {DIFF }}$ \\
\hline 1 & 1.377 & 1.0239 & 0.4472 \\
10 & 1.378 & 1.0715 & 1.4153 \\
50 & 1.474 & 1.1588 & 3.1000 \\
100 & 1.638 & 1.2119 & 4.0806 \\
\hline
\end{tabular}

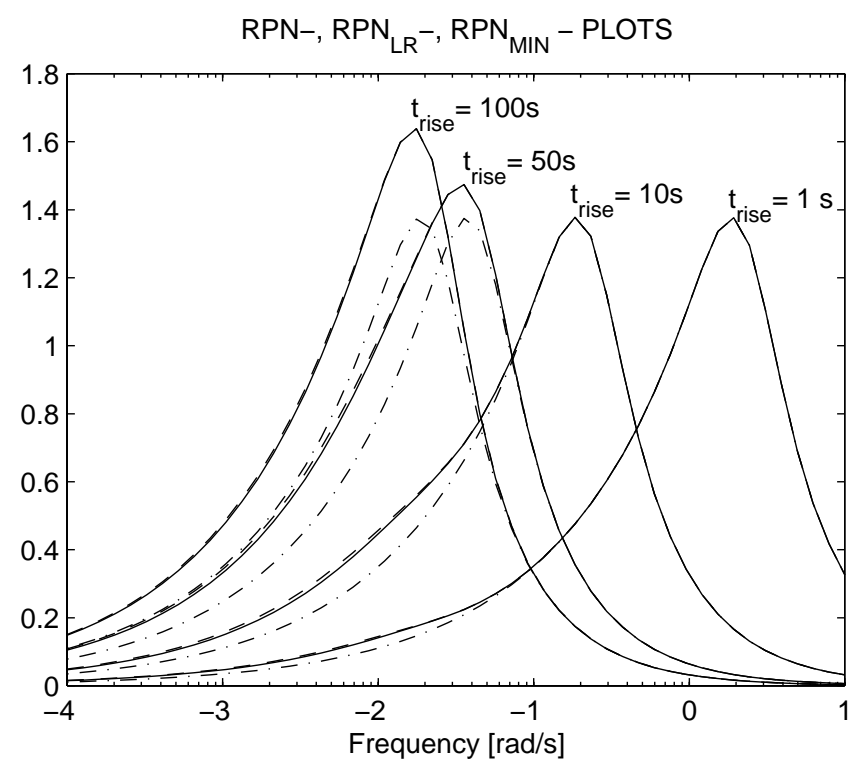

Figure 6: RPN plot (solid lines), $\mathrm{RPN}_{\mathrm{LR}}$ plot (dashed lines), and $\mathrm{RPN}_{\mathrm{MIN}}$ plot (dashdot lines) for MOP calculated using several rise times and 5\% overshoot.

Table 4: RPN indices for NMOP

\begin{tabular}{|l|l|l|l|}
\hline $\begin{array}{c}\text { Rise } \\
\text { Time [s] }\end{array}$ & \multicolumn{1}{|c|}{ RPN } & \multicolumn{1}{|c|}{ RPN $_{\text {RATIO }}$} & \multicolumn{1}{|c|}{ RPN $_{\text {DIFF }}$} \\
\hline 1 & 2.227 & 3.765 & 51.84 \\
10 & 2.265 & 2.679 & 33.22 \\
50 & 2.411 & 2.135 & 22.17 \\
100 & 2.498 & 1.952 & 18.33 \\
\hline
\end{tabular}




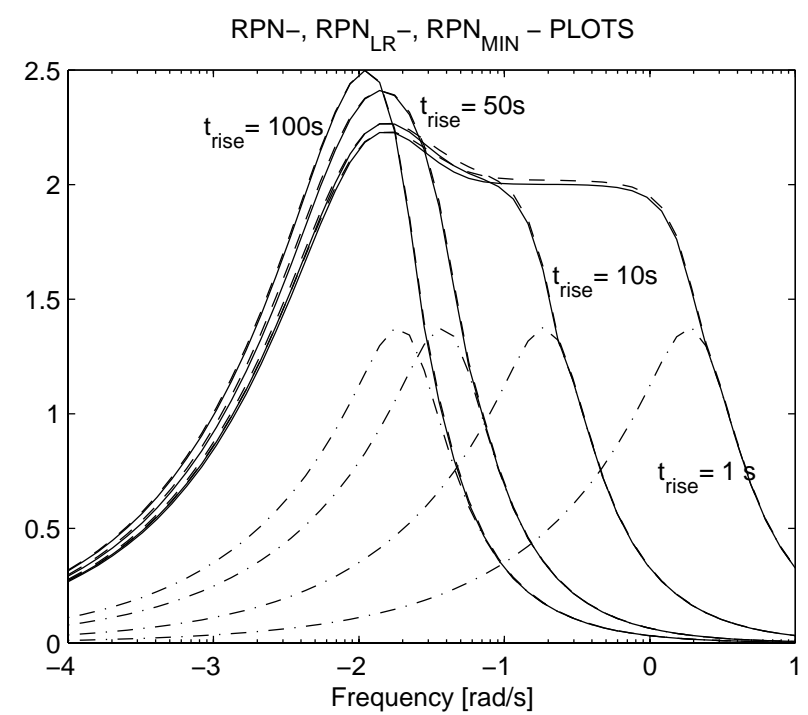

Figure 7: RPN plot (solid lines), $\mathrm{RPN}_{\mathrm{LR}}$ plot (dashed lines), and $\mathrm{RPN}_{\mathrm{MIN}}$ plot (dashdot lines) for MOP calculated using several rise times and 5\% overshoot.

It is important to mention that the RPN does not give a clear idea of the control difficulties for either OP. But on the other hand, RPN $\mathrm{NATIO}_{\text {and }} \mathrm{RPN}_{\mathrm{DIFF}}$ can do this very well. The closer $\mathrm{RPN}_{\mathrm{RATIO}}$ and $\mathrm{RPN}_{\mathrm{DIFF}}$ are to 1 and 0 , respectively, the more realizable the desired performance is.

Usually one is interested in using simple loworder controllers. When the system's directionality varies strongly with frequency, a higher order controller must be used. To determine how strong this dependence is, we use the $\mathrm{RPN}_{\mathrm{LR}}$ plot. Small differences between $\mathrm{RPN}_{\mathrm{LR}}$ and RPN plots indicate that a low-order controller will probably produce good results. The crossover frequency range (i.e., the region of the RPN peak) is especially important in this analysis. The dashed lines in Figures 6 and 7 correspond to the $\mathrm{RPN}_{\mathrm{LR}}$ plots. It is very difficult to distinguish them from the RPN plots. Therefore, we can conclude that good performance can be achieved by a low-order controller. In fact, increasing the controller order will not provide any improvement in control.

\section{VERIFICATION OF THE PREDICTIONS BY CLOSED-LOOP SIMULATIONS}

The controllers used in the simulations in Figures 8,9 , and 10 were obtained by applying the frequency response approximation method described in Trierweiler et al. (2000) and Engell and Müller (1993) to the optimally RPN-scaled system (see section RPN-Scaling Procedure). The specified closed-loop responses used in the controller design correspond to the same attainable performances used to calculate the RPN curves.
The simulations confirm the predictions made by RPN, RPN $\mathrm{LR}_{\text {L }}$, RPN $\mathrm{RATIO}_{\text {, and }} \mathrm{RPN}_{\text {DIFF. Figure } 8}$ shows that for MOP faster responses produce an almost decoupled setpoint change. For this OP, the only restriction on attainable performance is the power of the control action. If the control action is not fast enough, the levels of the tank start interacting with each other. This behavior has already been predicted by the dynamic RGA (cf. Figure 5).

Figures 9 and 10 show the closed-loop simulation for NMOP. In these figures, first the setpoint is changed in the worst possible direction, which corresponds to the output RHP zero direction $\left(\mathrm{y}_{\mathrm{z}}\right)$, and the disturbance rejection capacity is tested against the worst possible direction, which is given by the input RHP zero direction $\left(\mathrm{u}_{\mathrm{z}}\right)$. Both $\mathrm{y}_{\mathrm{z}}$ and $\mathrm{u}_{\mathrm{z}}$ are given in Table 2 . Figure 9 clearly shows that it is not possible to have a rise time faster than $100 \mathrm{~s}$. Here the RHP zero at 0.0128 restricts the attainable performance of the closed loop. Figure 10 analyzes the effect of the controller structure (i.e., full or decentralized) and order (i.e., PI or PID) in the performance of the closed loop. This result confirms our prediction that increasing the controller order does not improve the closed-loop performance for the quadruple-tank system.

To simplify the comparison between the attainable performances of the MOP and the NMOP, Figure 11 shows the simulation results obtained by the MOP with a decentralized PI controller and by the NMPO with a full PI controller. Note that MOP can be more than 10 times faster than NMOP.

The RPN methodology is also applied to tune MPC (Trierweiler et al., 2001) and multivariable controllers in general. All these methods are implemented in the RPN Toolbox (Farina, 2000). 

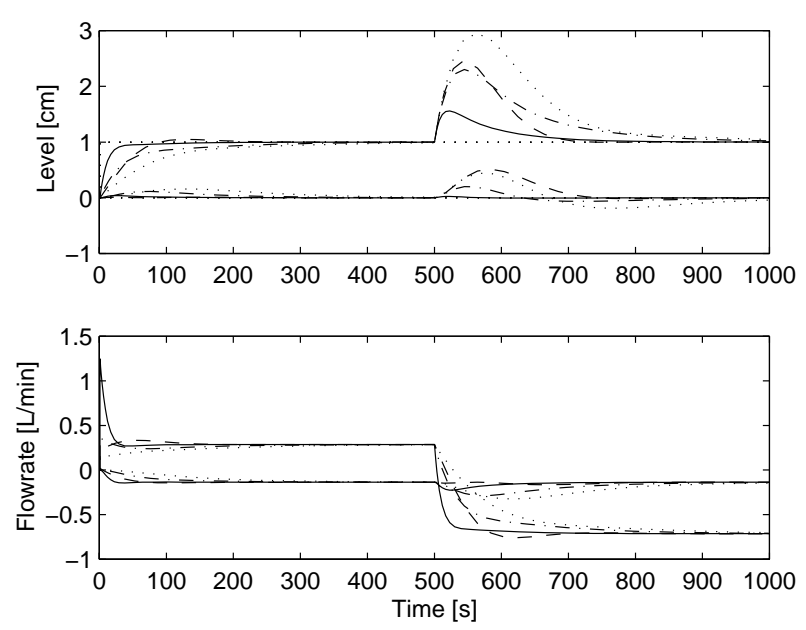

Figure 8: Setpoint change in $\mathrm{h}_{1}$ and disturbance rejection capacity for MOP: decentralized PI controller with $10 \mathrm{~s}$ rise time (solid line), full PI controller with $50 \mathrm{~s}$ rise time (dashed line), decentralized PI controller with $50 \mathrm{~s}$ rise time (dashdot line), and decentralized PI controller with $100 \mathrm{~s}$ rise time (dotted line).
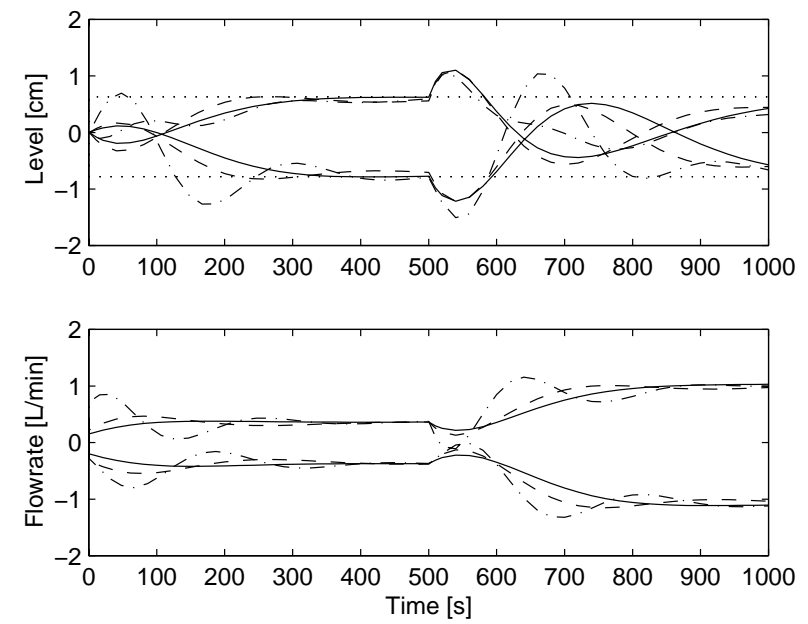

Figure 9: Setpoint change and disturbance rejection capacity for NMOP using full PI controller with $100 \mathrm{~s}$ (solid line), $50 \mathrm{~s}$ (dashdot line), and $10 \mathrm{~s}$ (dashed line) rise time.
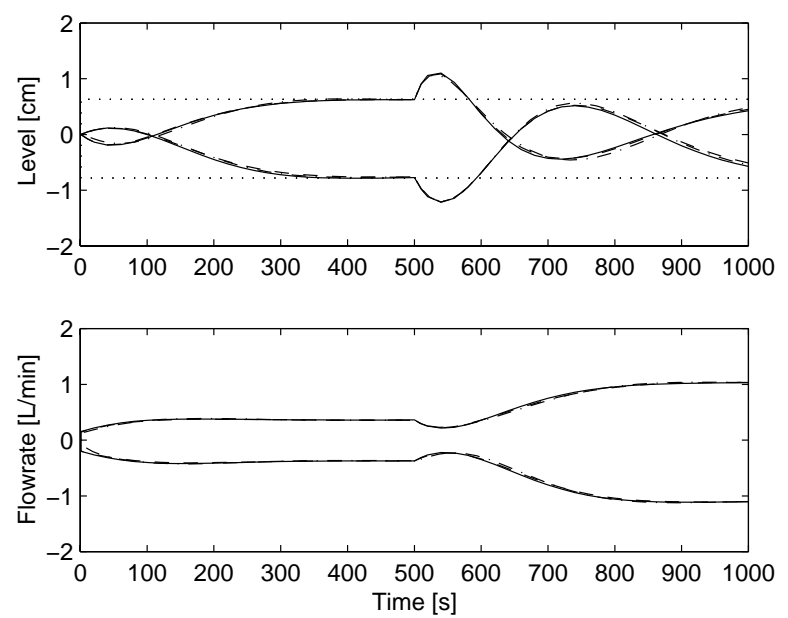

Figure 10: Setpoint change and disturbance rejection capacity for NMOP: full PI controller (solid line), decentralized PI controller (dashdot line), and decentralized PID controller (dashed line). All controllers were designed for a $100 \mathrm{~s}$ rise time. 

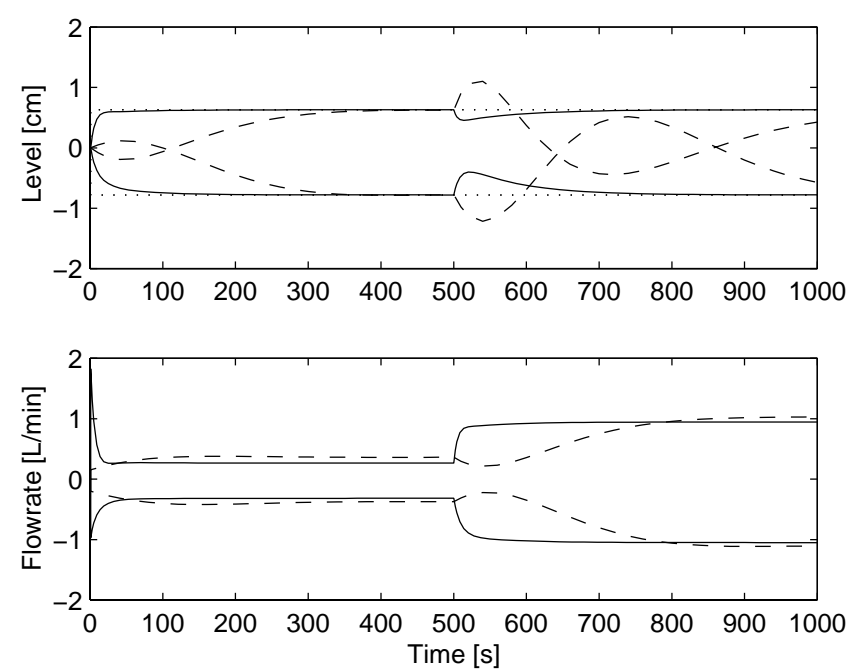

Figure 11: Setpoint change and disturbance rejection capacity for MOP with decentralized PI controller with $10 \mathrm{~s}$ rise time (solid line) and for NMOP with full PI controller with $100 \mathrm{~s}$ rise time (dashed line).

\section{CONCLUSIONS}

This paper demonstrated the application of the RPN concept in the IO-controllability analysis of the quadruple-tank system. It is shown that the RHP transmission zero put fundamental limitations on the performance of the system. Because of its dependence on attainable closed-loop performance, the RPN takes the effect of nonminimum-phase behavior and the desired performance of the closed loop into account. In addition, the frequencydependent directionality of the system is quantified correctly. Based solely on RPN, it is not possible to differentiate the effect of the desired performance on the closed-loop response, but using two new indices, $\mathrm{RPN}_{\mathrm{RATIO}}$ and $\mathrm{RPN}_{\mathrm{DIFF}}$, it is possible to see clearly how realizable a given desired performance is.

The analysis was performed using a linear nominal model, but it can be extended to include nonlinearities and uncertainties, as shown in Trierweiler (1997) and Trierweiler and Engell (1997b). In a subsequent step, structures with small RPN values can be analyzed further by the RPPN criterion or by a nonlinear simulation with a linear controller. RPPN (robust performance number of a plant set) is an extension of RPN and it is influenced by both the plant nonlinearities and the plant uncertainties. For the quadruple-tank system, the nonlinear analysis just confirms the conclusions and results obtained with simple linearized models, but for strong nonlinear systems, the nonlinear analysis must be performed.

\section{ACKNOWLEDGMENT}

The author would like to thank professors Míriam Tvrzska de Gouvea and Darci Odloak for inviting him to participate in the " $1^{\text {a }}$ Workshop em Integração de Processos Contínuos em Tempo Real", São Paulo (SP), November 23-24. This work was presented at that workshop. Additionally, the author would like to thank Prof. Míriam Tvrzska de Gouvea for the complete workshop organization and FAPESP for funding his attendance at the Workshop.

\section{REFERENCES}

Chiang R.Y. and M.G. Safonov (1992). Robust Control Toolbox. The MathWorks, Inc., Natick, MA.

Engell, S. and R. Müller (1993). Multivariable Controller Design by Frequency Response Approximation. Proc. 2nd Eur. Control Conf., pp. 1715-1720.

Farina, L. A. (2000). RPN-Toolbox: Uma Ferramenta para a Seleção de Estruturas de Controle, Master's thesis, Universidade Federal do Rio Grande do Sul (http://www.enq.ufrgs.br/rpn).

Havre, K. and S. Skogestad (1996). Effect of RHP Zeros and Poles on Performance in Multivariable Systems. Proc. Conf. CONTROL'96, Exeter, pp. 930-935.

Johansson, K. H. (2000). The Quadruple-Tank Process: A Multivariable Laboratory Process 
with an Ajustable Zero. IEEE Transactions on Control Systems Technology, Vol. 8(3), pp. 456465.

Skogestad, S. and I. Postlethwaite (1996). Multivariable Feedback Control Analysis \& Design. John Wiley\&Sons.

Trierweiler, J. O., Müller, R., and Engell, S. (2000). Multivariable Low Order Structured-Controller Design by Frequency Response Approximation. Brazilian Journal of Chemical Engineering, vol. 17, no. 04, pp.793-807.

Trierweiler, J. O., Farina, L. A., and Duraisk, R. G. (2001). RPN Tuning Strategy for Model Predictive Control. Preprints of DYCOPS-2001, pp. 283-288.
Trierweiler, J. O. and S. Engell (2000). A Case Study for Control Structure Selection: Air Separation Plant. Journal of Process Control, vol. 10 , pp. $237-243$.

Trierweiler, J.O. and S. Engell (1997a). The Robust Performance Number: A New Tool for Control Structure Design. Comp. Chem. Eng. (21), Suppl., pp. S409-414.

Trierweiler, J.O. and S. Engell (1997b). Controllability Analysis via the RPN for a CSTR with Van de Vusse Reaction, Proc. Eur. Control Conf. (ECC)'97, 1997, TU-A-H3.

Trierweiler, J.O. (1997). A Systematic Approach to Control Structure Design. Ph.D. Thesis, Univ. of Dortmund. 